\title{
Role of Phase Nanosegregation in the Photoluminescence Spectra of Halide Perovskites
}

\author{
Alessia Di Vito,* Alessandro Pecchia, Matthias Auf der Maur, Valerio Campanari, Faustino Martelli, \\ and Aldo Di Carlo
}

Cite This: J. Phys. Chem. Lett. 2021, 12, 11659-11665

Read Online

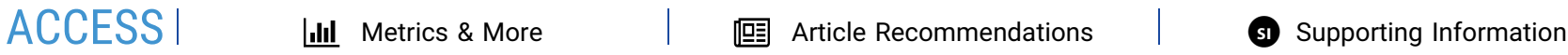

ABSTRACT: The study of $\mathrm{MAPbI}_{3}$ phase transitions based on temperature-dependent optical spectroscopy has recently gained a huge attention. Photoluminescence (PL) investigations of the tetragonal-orthorhombic transition suggest that tetragonal nanodomains are present below the transition temperature and signatures associated with tetragonal segregations are observed. We have studied the impact of phase nanosegregation across the orthorhombic-tetragonal phase transition of $\mathrm{MAPbI}_{3}$ on the system's properties employing a tight binding (TB) approach. The particle swarm optimization has been used to obtain a consistent set of TB parameters, where the target properties of the system have been derived by first-principles calculations. The theoretical results have been compared with the measured PL spectra for a temperature range going from 10 to $100 \mathrm{~K}$. Our model effectively captures the carriers' localization phenomenon induced by the presence of residual tetragonal nanodomains and demonstrates that the assumption of phase nanosegregation can explain the low-energy features in the PL spectra of $\mathrm{MAPbI}_{3}$.

$\mathrm{T}$ he major advance in the field of photovoltaics of the past decade has been represented by hybrid halide perovskites. These materials have general formula $\mathrm{AMX}_{3}$, where $\mathrm{A}$ is cesium, methylammonium or formamidinium, $\mathrm{M}$ is tin or lead, and $\mathrm{X}$ is chlorine, bromine, or iodine. Hybrid lead halide perovskites have been initially studied in dye-sensitized solar cells, ${ }^{1}$ and in a few years, they have shown a stately efficiency improvement. $^{2-8}$ Perovskite materials have been successfully implemented also in a variety of optoelectronic applications, such as light-emitting devices ${ }^{9}$ and lasing. ${ }^{10}$ Their success is due not only to their set of impressive electronic properties ${ }^{2,11-16}$ but also to the possibility of using low-cost techniques for their synthesis and deposition, such as solution-based methods. ${ }^{5}$

In spite of this success, there are still many open questions on the basic properties of this class of materials. In particular, hybrid perovskites show signs of phase transitions that take place in a temperature range of interest for technological applications. Specifically, for the prototypical methylammonium lead iodide perovskite $\left(\mathrm{MAPbI}_{3}\right)$, X-ray diffraction analysis $^{16,17}$ and calorimetric measurements ${ }^{18,19}$ reported the occurrence of two phase transitions at 162 and $327 \mathrm{~K}$, which are known to influence its optical properties. ${ }^{20-23}$ Poglitsch and Weber assigned the low-temperature transition to a change from an orthorhombic-to-tetragonal crystalline structure, and the high-temperature one to a change from a tetragonal-tocubic structure. ${ }^{17}$ Different behaviors have been reported for the orthorhombic-to-tetragonal phase transition of single crystal and thin film perovskites. Concerning thin films, the transition is observed at lower temperatures, and it involves a wider temperature range, ${ }^{24}$ depending on aspects such as strain, disorder, or polycrystallinity. In this case, the presence of tetragonal phase far below $160 \mathrm{~K}$ and down to $10 \mathrm{~K}$ was demonstrated. ${ }^{20,25-27}$ In the case of single crystals, scattering studies report that the phase transition occurs in a small temperature range. ${ }^{28,29}$

However, recent studies based on temperature-dependent optical spectroscopy exhibit the presence of different peaks and shoulders in the photoluminescence (PL) spectra of $\mathrm{MAPbI}_{3}$ single crystals. Such PL features are observed at lower temperatures with respect to the orthorhombic-tetragonal phase transition temperature. ${ }^{20,28,30}$ Schötz et al. investigated these additional peaks for pure tetragonal and orthorhombic phases. ${ }^{31}$ Furthermore, they analyzed the tetragonal-orthorhombic phase transition of a $\mathrm{MAPbI}_{3}$ single crystal employing temperature-dependent PL spectroscopy. ${ }^{32}$ They found that $0.015 \%$ of residual tetragonal phase is observed at $150 \mathrm{~K}$. Specifically, they estimated that the size of the tetragonal phase segregations is approximately $7-15 \mathrm{~nm}$ at $120 \mathrm{~K}$.

Thus, it is paramount for the success of perovskite materials to understand the relationship between the crystal structure of perovskite and its electronic and optical properties. In

Received: October 14, 2021

Accepted: November 15, 2021

Published: November 26, 2021 
theoretical studies related to halide perovskites, the $\mathrm{MAPbI}_{3}$ system has been widely used as prototypical model. ${ }^{33}$ Several challenges have to be tackled in order to successfully model the optical properties of such phase segregated nanodomains. The first problem is related to modeling of the individual bulk structures of orthorhombic and tetragonal phases and the matching of the two crystalline structures in the polycrystalline system. A clear issue in this context is that density functional calculations are out of reach for systems with hundreds of thousands of atoms; hence, accurate empirical methods are needed. The second related problem is how to obtain reliable electronic states of the nanodomains. A connected issue is how to set the correct band alignment between the tetragonal and the orthorhombic phases.

Our model is based on several ingredients, pivoting around a tight binding (TB) calculation of the electronic structure and optical transitions. TB was chosen because it intrinsically takes into account for the atomic details of the structures, in contrast to envelop function methods (e.g., $k \cdot p$ ). However, TB requires a careful construction of the atomic model at the orthorhombic/tetragonal interfaces in order to avoid artifacts. In particular, the TB parameters needs to be exportable across different phases and the effect of bond distortion at the interfaces needs to be taken into account. This goal has been achieved by constructing a fully consistent parametrization, able to reproduce the density functional theory (DFT) bulk band structures of orthorhombic, tetragonal, and pseudocubic phases of halide perovskites. The cubic phase was added in order to increase the amount of constrains in the fitting procedure and obtain a parametrization valid throughout the whole temperature scale. This approach, together with proper cross checks on strained crystals allowed to obtain a consistent set, valid also at the interfaces, where bonds are significantly distorted.

In order to construct atomistic models of the tetragonal nanocrystals embedded in the orthorhombic matrix, we have developed a new scheme within the TiberCAD atomistic structure generator, ${ }^{34}$ now able to fill different spatial regions with different atomic crystalline structures and gluing them together with a correct bond-map. This approach is rather in contrast to the usual construction of pseudomorphic crystalline structures that share the same unit cells (like in III-V heterostructures).

The band-edge discontinuity at the interfaces is another parameter needed in TB calculations. Since a reliable value for the interfaces of interest in the present work is not directly available in the literature, we have computed it using a DFT approach. We should remark that DFT, being a ground-state theory, can only provide the valence-band (VB) discontinuity, whereas the energy gaps are fixed from experimental values and the conduction band discontinuity follows as a consequence.

Here, we study the role of phase nanosegregation across the orthorhombic-tetragonal phase transition of $\mathrm{MAPbI}_{3}$ on the electronic and optical properties of the system. We simulate the presence of tetragonal nanodomains within the orthorhombic phase. The spatial localization of carriers, induced by the increasing dimension of the tetragonal domains, and its impact on the emission properties of perovskite are analyzed. The theoretical results are compared with the measured PL spectra for a temperature range going from 10 to $100 \mathrm{~K}$.

In order to describe the atomic scale features of the system, we employed the $\mathrm{TB}$ module of the TiberCAD software package. ${ }^{34}$ Since the TB method is not designed to yield the optimized crystal structure, a DFT calculation has been employed to relax the atomic positions. DFT simulations have been performed at the GGA level of approximation using the Quantum Espresso package ${ }^{35}$ with scalar-relativistic PBE pseudopotentials. ${ }^{36}$ The kinetic energy cutoff has been set to $25 \mathrm{Ry}$ for wave functions and $200 \mathrm{Ry}$ for charge density and potential, and we used a $4 \times 4 \times 4 k$-points grid for reciprocal space integration. It has been demonstrated that the electronic structure of $\mathrm{MAPbI}_{3}$ can be reproduced with sufficient accuracy employing the minimal $\mathrm{sp}^{3}$ TB basis set. ${ }^{37}$ Thus, we followed the prescription of Boyer-Richard et al. ${ }^{37}$ for the choice of TB basis. Furthermore, DFT calculations, investigating the specific contribution of each atomic species to the electronic states around the band gap, highlight that the weight associated with the MA cation is not relevant. ${ }^{38}$ Based on the mentioned results, our TB model includes only the $\mathrm{Pb}-\mathrm{I}$ cage and does not include the organic cation. In order to derive the theoretical optical spectra, the momentum matrix elements associated with each optical transition are needed. These are calculated from the electron and hole states evaluated with the TB approach. We considered the first 12 electron and the first 12 hole states, and the $G, X, Z, M, R$, and $A$ high-symmetry points of the Brillouin zone for reciprocal space integration. The phase-segregated system is modeled placing a tetragonal domain, with nanometric dimensions, at the center of an orthorhombic box, with a fixed side length of $25 \mathrm{~nm}$. According to the estimate presented in the study of Schötz et al., ${ }^{32}$ we considered tetragonal nanodomains with dimensions of 5, 9, and $14 \mathrm{~nm}$.

The TB Hamiltonian matrix elements (HME) are derived as fitting parameters able to reproduce the target DFT band structure. In order to optimize the fitting procedure, we used the particle swarm optimization (PSO) algorithm. ${ }^{40}$ Note that the optimization of the TB parameters is performed taking into account the band structure of all the three $\mathrm{MAPbI}_{3}$ phases simultaneously. Moreover, the experimental band gap value has been taken into account as target feature, adjusting the DFT energy gap to the measured one. ${ }^{33}$ This is discussed in detail in Section I of the Supporting Information. In parts a and b of Figure 1, the TB band structures of, respectively, orthorhombic and tetragonal $\mathrm{MAPbI}_{3}$ obtained after the $\mathrm{PSO}$ are shown. The related energy gap values are listed in Table 1 . Our results are compared with the measurements and GW calculations found in the reference study of Quarti et al., ${ }^{33}$ also reported in the table. The orthorhombic energy gap value derived by our TB model is consistent with the reference GW calculation and both approaches overestimate the experimental result. Nevertheless, the energy gap difference between tetragonal and orthorhombic $\mathrm{MAPbI}_{3}$ obtained with TB is in agreement with measurements. Since we are interested in simulating the presence of tetragonal domains below the phase transition temperature and its effect on the emission properties of $\mathrm{MAPbI}_{3}$, a consistent energy gap difference between the two crystals is paramount to properly describe the confinement of carriers and the related spatial localization. For completeness, the effective masses for holes and electrons obtained by parabolic fitting of the TB valence and conduction band edge for tetragonal $\mathrm{MAPbI}_{3}$ along the directions $\Gamma \rightarrow X$ and $\Gamma \rightarrow Z$ are reported in Table 2. Our results are compared with the theoretical values obtained by Umari et al. ${ }^{39}$

Finally, the valence band offset between the two crystal structures is obtained by DFT calculations, following the steps described in the reference work of Weston et al. ${ }^{41}$ The position 


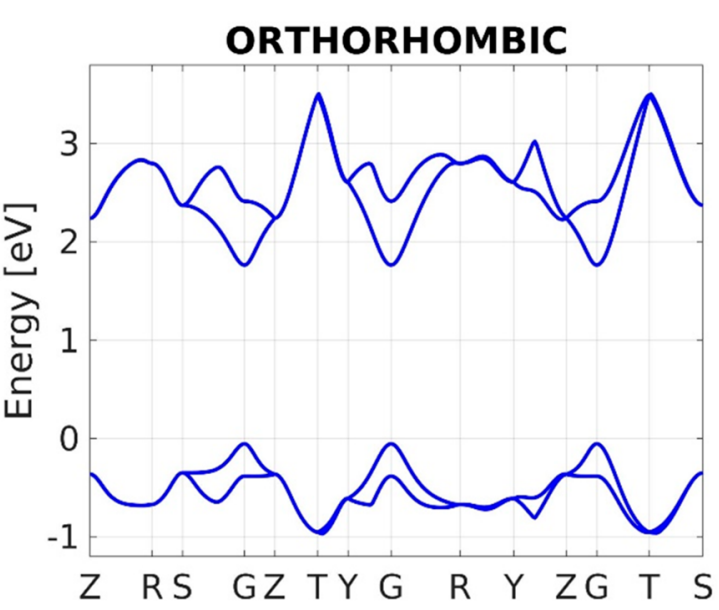

a)

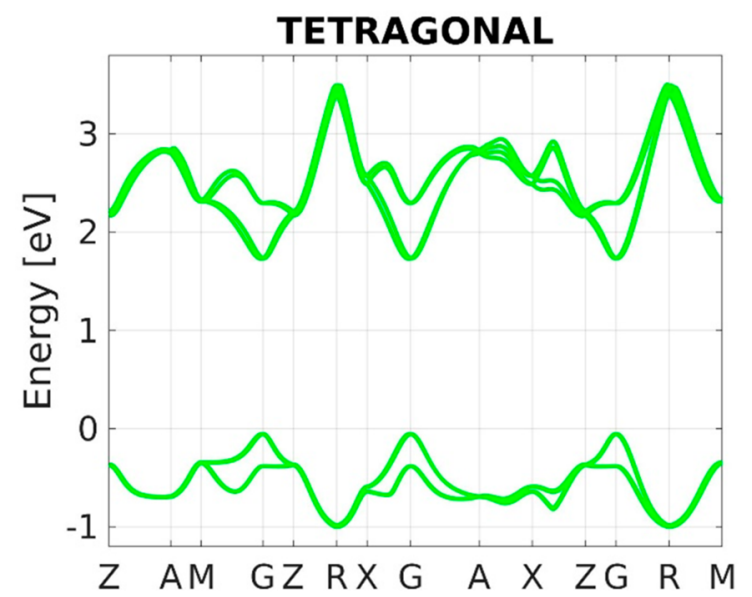

b)

Figure 1. TB band structure of (a) orthorhombic and (b) tetragonal $\mathrm{MAPbI}_{3}$ derived after PSO.

Table 1. Energy Gap Values (eV) for Orthorhombic and Tetragonal $\mathrm{MAPbI}_{3}$

\begin{tabular}{lcc} 
& orthorhombic & tetragonal \\
TB (after PSO) & 1.82 & 1.78 \\
DFT-GW & 1.81 & 1.67 \\
experiment $^{33}$ & 1.65 & 1.61 \\
\hline
\end{tabular}

Table 2. Effective Masses for Holes and Electrons of Tetragonal $\mathrm{MAPbI}_{3}$ along the Directions $\Gamma \rightarrow X$ and $\Gamma \rightarrow Z$

\begin{tabular}{lccc} 
& TB (after PSO) & SOC-DFT $^{39}$ & SOC-GW $^{39}$ \\
$m_{e, \Gamma \rightarrow X}$ & 0.1 & 0.16 & 0.17 \\
$m(h, \Gamma \rightarrow X$ & 0.13 & 0.22 & 0.20 \\
$m_{e, \Gamma \rightarrow Z}$ & 0.3 & 0.26 & 0.29 \\
$m_{e, \Gamma \rightarrow Z}$ & 0.4 & 0.44 & 0.40 \\
\hline
\end{tabular}

of the valence band maximum (VBM) with respect to the average electrostatic potential is determined from the DFT bulk calculations, separately for each crystal. Then, the alignment of the average electrostatic potential between the two crystals is determined from a supercell calculation, analyzing the average of the electrostatic potential in the bulk-like region of each crystal. Concerning the supercell calculation, we considered the $x-y$ plane-averaged electrostatic potential integrated along the $z$ direction over the length of the unit cell. The calculated data exhibit the superposition of a polarization potential, originating from the heterojunction between the two crystals, and a step-like potential, representing the electrostatic potential difference between the bulk-like regions of the two crystals. The computational details are reported in Section II of the Supporting Information. Combining the information from bulk calculations, i.e., the position of the VBM with respect to the mean electrostatic potential for the two separate bulk materials, with the electrostatic potential difference derived by superlattice calculations, we obtain the valence band alignment reported in Figure 2. The valence band offset between tetragonal and

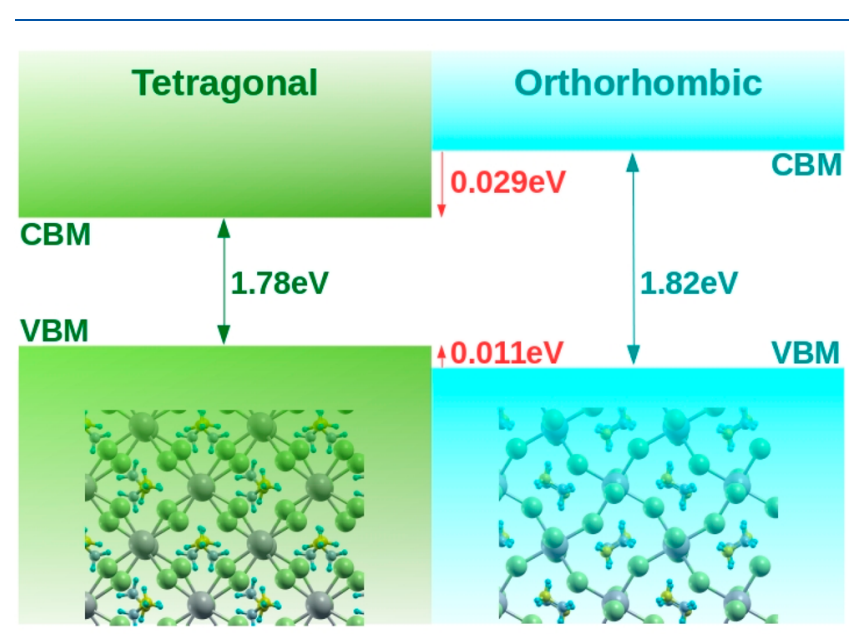

Figure 2. Band alignments between tetragonal and orthorhombic $\mathrm{MAPbI}_{3}$ derived by the combination of bulk and superlattice DFT calculations. ${ }^{41}$ The conduction band discontinuity is obtained considering the TB band gap values.

orthorhombic $\mathrm{MAPbI}_{3}$ is $0.011 \mathrm{eV}$, thus, the conduction band discontinuity is equal to $0.029 \mathrm{eV}$, i.e., the band gap difference between the two materials minus the valence band discontinuity value.

In Figure 3a, the conduction band (CB) and VB states of the system are reported for the considered tetragonal domain dimensions. We can see that the energy gap is significantly reduced for the $14 \mathrm{~nm}$ tetragonal domain system, as well as the value of the $\mathrm{CB}$ minimum (CBM), while the difference in VBM, CBM, and energy gap values between the $5 \mathrm{~nm}$ and the $9 \mathrm{~nm}$ tetragonal domain system is not relevant. On the other hand, the VBM value slightly increases for the largest domain size. In fact, the domain size dependence of the energy gap is almost equal to the CBM behavior. These trends originate from the spatially localized ground state electron wave function within the tetragonal domain. In fact, the $14 \mathrm{~nm}$ tetragonal segregation is sufficiently large to induce spatial localization of carriers. This phenomenon is clearly represented in panel b of Figure 3, where the isosurface containing the $50 \%$ of the total ground state density of the electron is depicted for the $14 \mathrm{~nm}$ tetragonal nanodomain. On the contrary, in panel c of Figure 3 , where the same quantity is shown for the $5 \mathrm{~nm}$ tetragonal segregation, we can see that the spatial localization of the ground state electron wave function is not observed, even if the isosurface in panel c contains only the $10 \%$ of the total ground state density. Furthermore, as the conduction band discontinuity is much more pronounced than the valence band one, we 

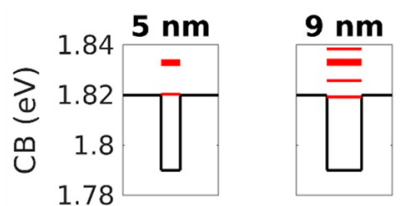

$14 \mathrm{~nm}$
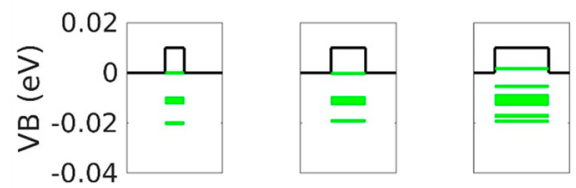

a)

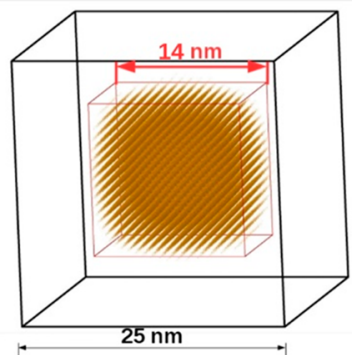

b)

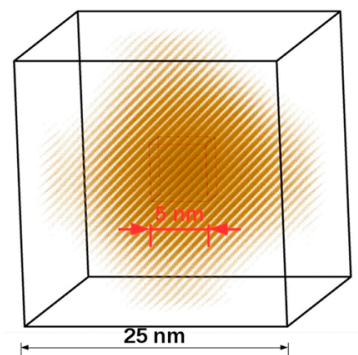

c)
Figure 3. (a) Bulk band edge profiles across the considered tetragonal domains and energies of the valence (green) and conduction (red) band states at the gamma point. (b) Ground state electron wave function for the $14 \mathrm{~nm}$ tetragonal domain system. The isosurfaces containing $50 \%$ of the total ground state density are shown. (c) Ground state electron wave function for the $5 \mathrm{~nm}$ tetragonal domain system. The isosurfaces containing $10 \%$ of the total ground state density are shown.

do not expect a significant localization of the ground state hole wave function. In fact, the VBM value is almost unaffected by the dimension of the tetragonal segregation.

In Figure 4, we show how the emission spectrum varies when the dimension of the tetragonal nanosegregation is increased from 5 to $14 \mathrm{~nm}$. The three spectra are calculated at $150 \mathrm{~K}$ and normalized to unity. As expected from the behavior of the energy gap discussed above, we can see in the figure that,

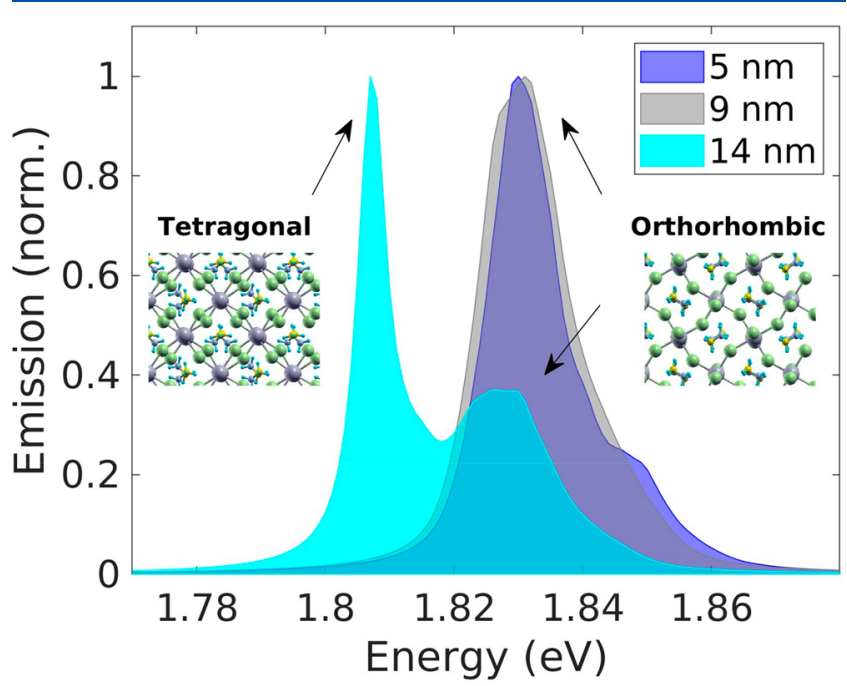

Figure 4. Emission spectra calculated at $150 \mathrm{~K}$ for the three considered tetragonal domain dimensions. only for the $14 \mathrm{~nm}$ tetragonal segregation, the red-shift of the peak emission energy is observed. Furthermore, in the emission spectrum of the system with the highest dimension of the tetragonal domain, depicted by the cyan area plot in Figure 4, both the low-energy and the high-energy peaks, respectively associated with the tetragonal and the orthorhombic crystal structures, are visible. The low-energy feature in the calculated spectrum is due to the contribution of the electron ground state localized within the low-band gap tetragonal crystal, while the high-energy feature originates from higher-energy states coming from the prevalent orthorhombic phase. As discussed for Figure 3, the $5 \mathrm{~nm}$ and the $9 \mathrm{~nm}$ tetragonal segregations are not sufficiently large to yield a spatially localized electron ground state, thus, for these systems, the low-energy peak is not observed in the calculated spectra. The results obtained with different values of the bandoffset are reported in Section III of the Supporting Information, for completeness.

A consistent description of the emission properties of the system can be obtained as a superposition of the calculated spectra for the minimum and maximum tetragonal domain dimension, i.e., $x E_{5 n m}+(1-x) E_{14 n m}$, where $E_{5 n m}$ and $E_{14 n m}$ are the calculated emission spectra for the 5 and $14 \mathrm{~nm}$ domain size, respectively, and $x$ is a temperature related weighting parameter derived by fitting the measured spectra. The results are represented in parts a-d of Figure 5, where panels a and c show the experimental data obtained by PL measurements in a temperature range going from 10 to $100 \mathrm{~K}$ with incident power density of 1.3 and $13 \mathrm{~W} / \mathrm{cm}^{2}$, respectively, and the related fitted spectra are shown in panels $b$ and $d$. In order to fit the experimental data, we have used a weighting parameter that is not a linear function of temperature. Thus, the emission properties of the system cannot be simply described assuming that the fraction of tetragonal phase decreases when the temperature is lowered. The behavior of the fitting parameter and the experimental setup for the PL measurements are discussed in Section IV of the Supporting Information. Note that the calculated spectra are blue-shifted with respect to the measured ones. This is due to the overestimation of the energy gap values, as discussed for Table 1. As it can be seen in Figure 5 , the low energy feature, originating from localized states confined within the tetragonal domain, is visible even far below the transition temperature, indicating that a significant fraction of tetragonal $\mathrm{MAPbI}_{3}$ is still present. ${ }^{27}$ Some differences can be found between experimental and fitted spectra about the width and the temperature trend of tetragonal peak. In experimental data the tetragonal peak appears wider and less sharp, moreover seems to perform a slight red-shift lowering temperature below $100 \mathrm{~K}$. These two features can be explained by the presence, in experimental data, of spectral components due to recombination on defect states lying within the band gap. Defect states related luminescence can appear in a wide spectral range and it tends to saturate increasing the temperature. Therefore, they can influence both the width and the position of other peaks. ${ }^{27}$ On the other hand, the redshift of the orthorhombic peak observed in panels $b$ and $d$ is qualitatively consistent with the measurements of panels a and c. In fact, the occupation of the high-energy states associated with the orthorhombic crystal for different temperatures is accounted for in the theoretical spectra. The occupation decreases when the temperature is lowered, yielding an overall red-shift of the orthorhombic peak. This effect is further discussed in Section IV of the Supporting Information. 


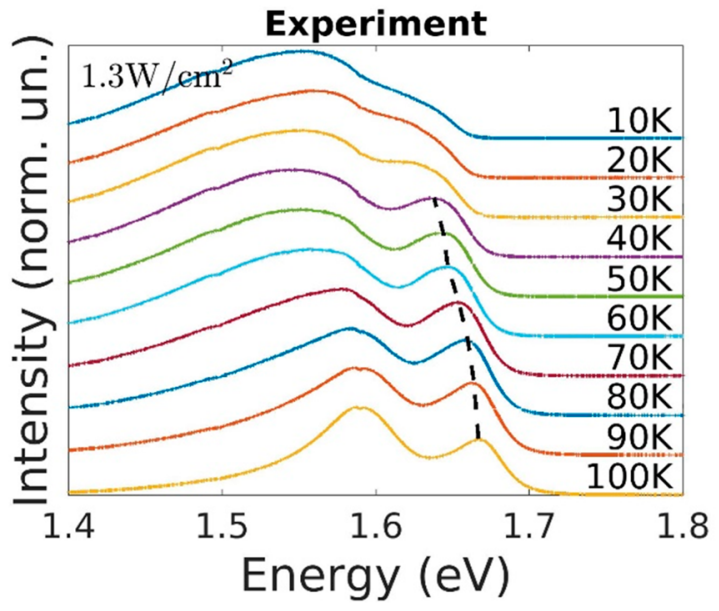

a)

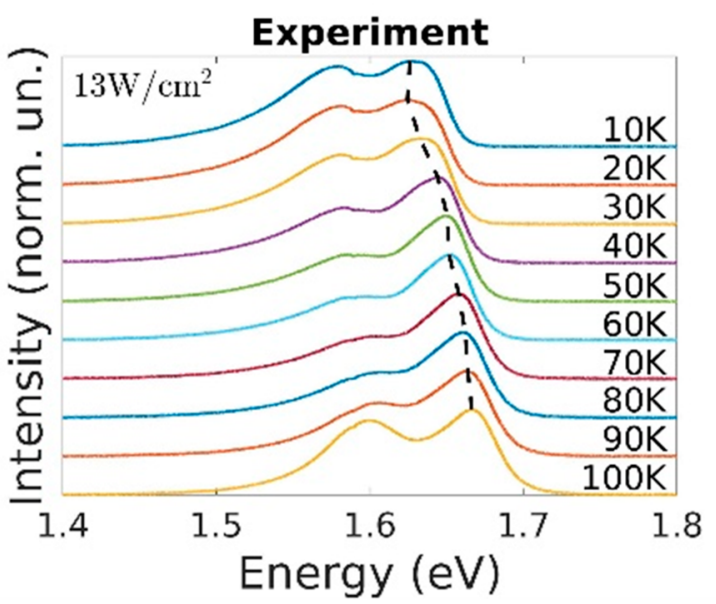

c)

Figure 5. Experimental spectra obtained by PL measurements in a temperature range going from 10 to $100 \mathrm{~K}$ with incident power density of a $1.3 \mathrm{~W} / \mathrm{cm}^{2}$ and $\mathrm{c} 13 \mathrm{~W} / \mathrm{cm}^{2}$. The related theoretical spectra are shown in panels $\mathrm{b}$ and $\mathrm{d}$, respectively. The higher density of excitation is taken into account using a carrier density ten times larger in panel $d$ with respect to panel $b$.

In conclusion, our simulations demonstrate that the presence of residual nanodomains of tetragonal phase below the tetragonal-orthorhombic transition temperature can explain the low-energy features in the $\mathrm{PL}$ spectra of $\mathrm{MAPbI}_{3}$ perovskite down to $10 \mathrm{~K}$. Even if our theoretical model does not address defect states recombination that can affect both the width and the position of PL peaks, the calculated spectra are consistent with the measured ones and the red-shift of the orthorhombic peak is confirmed. However, based on TB simulations and measurements, we think that the PL features cannot be quantitatively related to the exact fraction of residual tetragonal phase. In fact, the relative intensity of the tetragonal and orthorhombic peaks varies with the density of excitation employed in the experiment for a same value of temperature. Moreover, the fraction of tetragonal phase used in the theoretical spectra to fit the experimental trends is not a linear function of the temperature. Finally, the TB model, supported by PSO to determine the HME and by DFT calculations to estimate the VB offset, effectively capture the carriers' localization phenomenon at the origin of the low-energy PL features.

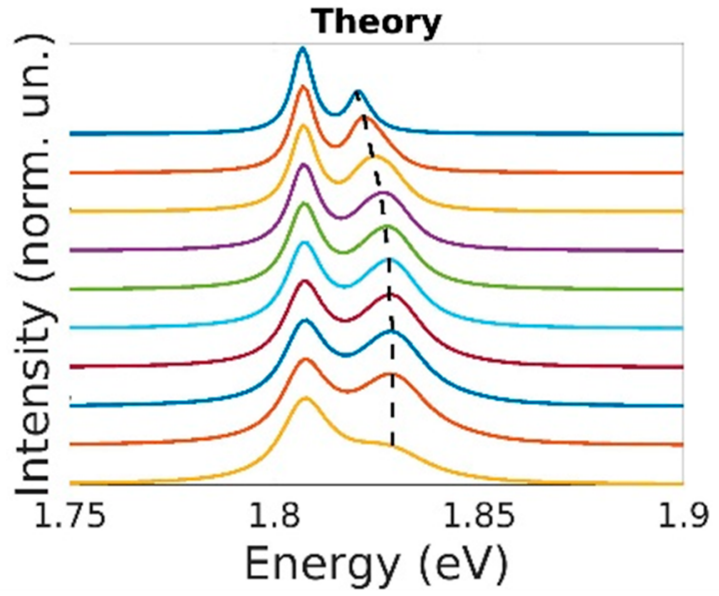

b)

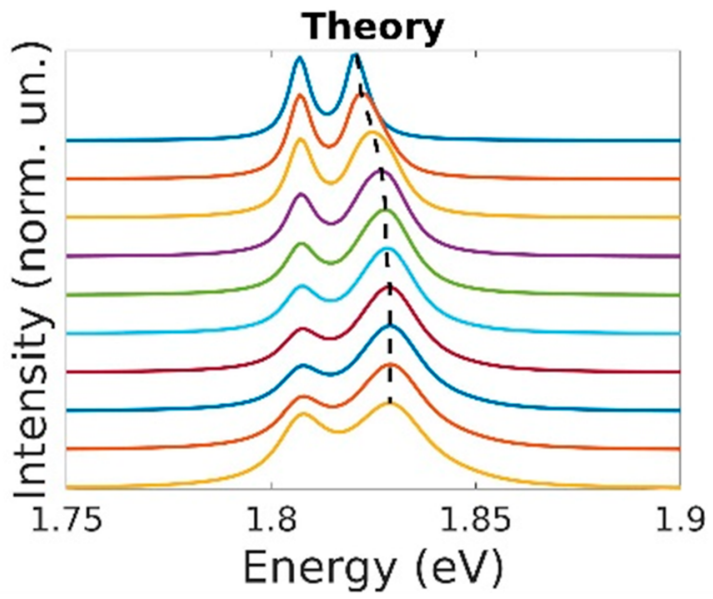

d)

\section{ASSOCIATED CONTENT}

Supporting Information

The Supporting Information is available free of charge at https://pubs.acs.org/doi/10.1021/acs.jpclett.1c03378.

Additional computational and experimental details, materials, and methods (PDF)

\section{AUTHOR INFORMATION}

\section{Corresponding Author}

Alessia Di Vito - University of Rome "Tor Vergata", 00133 Rome, Italy; o orcid.org/0000-0003-4774-3488;

Email: alessia.di.vito@uniroma2.it

\section{Authors}

Alessandro Pecchia - CNR-ISMN, 00014 Monterotondo Stazione, Rome, Italy

Matthias Auf der Maur - University of Rome "Tor Vergata", 00133 Rome, Italy

Valerio Campanari - University of Rome "Tor Vergata", 00133 Rome, Italy

Faustino Martelli - CNR-IMM, 00133 Rome, Italy; (1) orcid.org/0000-0002-4496-4165 
Aldo Di Carlo - University of Rome "Tor Vergata", 00133 Rome, Italy; LASE, Laboratory of Advanced Solar Energy, National University of Science and Technology "MISiS", 119049 Moscow, Russia; ○ orcid.org/0000-0001-68282380

Complete contact information is available at:

https://pubs.acs.org/10.1021/acs.jpclett.1c03378

\section{Notes}

The authors declare no competing financial interest.

\section{ACKNOWLEDGMENTS}

Valerio Campanari and Aldo Di Carlo acknowledge the support of the Italian Ministry of Economic Development in the framework of the Operating Agreement with ENEA for Research on the Electric System. Aldo Di Carlo gratefully acknowledges the Ministry of Education and Science of the Russian Federation in the framework of the Increase Competitiveness Program of NUST (MISiS) (No. K2-202027).

\section{REFERENCES}

(1) Kojima, A.; Teshima, K.; Shirai, Y.; Miyasaka, T. Organometal Halide Perovskites as Visible-light Sensitizers for Photovoltaic Cells. J. Am. Chem. Soc. 2009, 131, 6050-6051.

(2) Im, J.-H.; Lee, C.-R.; Lee, J.-W.; Park, S. W.; Park, N.-G. 6.5\% Efficient Perovskite Quantum-dot-sensitized Solar Cell. Nanoscale 2011, 3, 4088-4093.

(3) Lee, M. M.; Teuscher, J.; Miyasaka, T.; Murakami, T. N.; Snaith, H. J. Efficient Hybrid Solar Cells Based on Meso-superstructured Organometal Halide Perovskites. Science 2012, 338, 643-647.

(4) Burschka, J.; Pellet, N.; Moon, S.-J.; Humphry-Baker, R.; Gao, P.; Nazeeruddin, M. K.; Grätzel, M. Sequential Deposition as a Route to High-performance Perovskite-sensitized Solar Cells. Nature 2013, 499, 316-319.

(5) Liu, M.; Johnston, M. B.; Snaith, H. J. Efficient Planar Heterojunction Perovskite Solar Cells by Vapour Deposition. Nature 2013, 501, 395-398.

(6) Edri, E.; Kirmayer, S.; Cahen, D.; Hodes, G. High Open-circuit Voltage Solar Cells Based on Organic-inorganic Lead Bromide Perovskite. J. Phys. Chem. Lett. 2013, 4, 897-902.

(7) Ball, J. M.; Lee, M. M.; Hey, A.; Snaith, H. J. Low-temperature Processed Mesosuperstructured to Thin-film Perovskite Solar Cells. Energy Environ. Sci. 2013, 6, 1739-1743.

(8) Malinkiewicz, O.; Yella, A.; Lee, Y. H.; Espallargas, G. M.; Graetzel, M.; Nazeeruddin, M. K.; Bolink, H. J. Perovskite Solar Cells Employing Organic Charge-transport Layers. Nat. Photonics 2014, 8, $128-132$.

(9) Stranks, S. D.; Snaith, H. J. Metal-halide Perovskites for Photovoltaic and Light-emitting Devices. Nat. Nanotechnol. 2015, 10, 391-402.

(10) Zhu, H.; Fu, Y.; Meng, F.; Wu, X.; Gong, Z.; Ding, Q.; Gustafsson, M. V.; Trinh, M. T.; Jin, S.; Zhu, X. Y. Lead Halide Perovskite Nanowire Lasers with Low Lasing Thresholds and High Quality Factors. Nat. Mater. 2015, 14, 636-642.

(11) Edri, E.; Kirmayer, S.; Mukhopadhyay, S.; Gartsman, K.; Hodes, G.; Cahen, D. Elucidating the Charge Carrier Separation and Working Mechanism of $\mathrm{CH}_{3} \mathrm{NH}_{3} \mathrm{PbI}_{3-\mathrm{x}} \mathrm{Cl}_{\mathrm{x}}$ Perovskite Solar Cells. Nat. Commun. 2014, 5, 1-8.

(12) Edri, E.; Kirmayer, S.; Henning, A.; Mukhopadhyay, S.; Gartsman, K.; Rosenwaks, Y.; Hodes, G.; Cahen, D. Why Lead Methylammonium tri-Iodide Perovskite-based Solar Cells Require a Mesoporous Electron Transporting Scaffold (but not necessarily a hole conductor). Nano Lett. 2014, 14, 1000-1004.

(13) Stranks, S. D.; Eperon, G. E.; Grancini, G.; Menelaou, C.; Alcocer, M. J. P.; Leijtens, T.; Herz, L. M.; Petrozza, A.; Snaith, H. J.
Electron-hole Diffusion Lengths Exceeding 1 micrometer in an Organometal Trihalide Perovskite Absorber. Science 2013, 342, 341344.

(14) Xing, G.; Mathews, N.; Sun, S.; Lim, S. S.; Lam, Y. M.; Grätzel, M.; Mhaisalkar, S.; Sum, T. C. Long-range Balanced Electron-and Hole-transport Lengths in Organic-inorganic $\mathrm{CH}_{3} \mathrm{NH}_{3} \mathrm{PbI}_{3}$. Science 2013, 342, 344-347.

(15) Wehrenfennig, C.; Eperon, G. E.; Johnston, M. B.; Snaith, H. J.; Herz, L. M. High Charge Carrier Mobilities and Lifetimes in Organolead trihalide Perovskites. Adv. Mater. 2014, 26, 1584-1589.

(16) Stoumpos, C. C.; Malliakas, C. D.; Kanatzidis, M. G. Semiconducting Tin and Lead Iodide Perovskites with Organic Cations: Phase Transitions, High Mobilities, and Near-infrared Photoluminescent Properties. Inorg. Chem. 2013, 52, 9019-9038.

(17) Poglitsch, A.; Weber, D. Dynamic Disorder in Methylammonium Trihalogeno Plumbates (ii) Observed by Millimeter-wave Spectroscopy. J. Chem. Phys. 1987, 87, 6373-6378.

(18) Onoda-Yamamuro, N.; Matsuo, T.; Suga, H. Calorimetric and IR Spectroscopic Studies of Phase Transitions in Methylammonium Trihalogeno Plumbates (ii). J. Phys. Chem. Solids 1990, 51, 13831395.

(19) Knop, O.; Wasylishen, R. E.; White, M. A.; Cameron, T. S.; Van Oort, M. J. M. Alkylammonium Lead Halides. Part 2. $\mathrm{CH}_{3} \mathrm{NH}_{3} \mathrm{PbX}_{3}$ $(\mathrm{x}=\mathrm{Cl}, \mathrm{Br}, \mathrm{I})$ Perovskites: Cuboctahedral Halide Cages with Isotropic Cation Reorientation. Can. J. Chem. 1990, 68, 412-422.

(20) Kong, W.; Ye, Z.; Qi, Z.; Zhang, B.; Wang, M.; Rahimi-Iman, A.; $\mathrm{Wu}, \mathrm{H}$. Characterization of an Abnormal Photoluminescence Behavior upon Crystal-phase Transition of Perovskite $\mathrm{CH}_{3} \mathrm{NH}_{3} \mathrm{PbI}_{3}$. Phys. Chem. Chem. Phys. 2015, 17, 16405-16411.

(21) Glushkova, A.; Mantulnikovs, K.; Giriat, G.; Semeniuk, K.; Forró, L.; Horváth, E.; Arakcheeva, A. Effect of Thermal Cycling on the Structural Evolution of Methylammonium Lead Iodide Monitored around the Phase Transition Temperatures. Sol. RRL 2019, 3, 1900044.

(22) Singh, S.; Li, C.; Panzer, F.; Narasimhan, K. L.; Graeser, A.; Gujar, T. P.; Kohler, A.; Thelakkat, M.; Huettner, S.; Kabra, D. Effect of Thermal and Structural Disorder on the Electronic Structure of Hybrid Perovskite Semiconductor $\mathrm{CH}_{3} \mathrm{NH}_{3} \mathrm{PbI}_{3}$. J. Phys. Chem. Lett. 2016, 7, 3014-3021.

(23) Parrott, E. S.; Milot, R. L.; Stergiopoulos, T.; Snaith, H. J.; Johnston, M. B.; Herz, L. M. Effect of Structural Phase Transition on Charge-carrier Lifetimes and Defects in $\mathrm{CH}_{3} \mathrm{NH}_{3} \mathrm{SnI}_{3}$ Perovskite. J. Phys. Chem. Lett. 2016, 7, 1321-1326.

(24) Osherov, A.; Hutter, E. M.; Galkowski, K.; Brenes, R.; Maude, D. K.; Nicholas, R. J.; Plochocka, P.; Bulovíc, V.; Savenije, T. J.; Stranks, S. D. The Impact of Phase Retention on the Structural and Optoelectronic Properties of Metal Halide Perovskites. Adv. Mater. 2016, 28, 10757-10763.

(25) Wehrenfennig, C.; Liu, M.; Snaith, H. J.; Johnston, M. B.; Herz, L. M. Charge Carrier Recombination Channels in the LowTemperature Phase of Organic-inorganic Lead Halide Perovskite Thin Films. APL Mater. 2014, 2, 081513.

(26) Panzer, F.; Li, C.; Meier, T.; Köhler, A.; Huettner, S. Impact of Structural Dynamics on the Optical Properties of Methylammonium Lead Iodide Perovskites. Adv. Energy Mater. 2017, 7, 1700286.

(27) Campanari, V.; Agresti, A.; Pescetelli, S.; Sivan, A. K.; Catone, D.; O’Keeffe, P.; Turchini, S.; Di Carlo, A.; Martelli, F. Systematic Approach to the Study of the Photoluminescence of $\mathrm{MAPbI}_{3}$. Phys. Rev. Mater. 2021, 5, 035409.

(28) Fang, H.-H.; Raissa, R.; Abdu-Aguye, M.; Adjokatse, S.; Blake, G. R.; Even, J.; Loi, M. A. Photophysics of Organic-inorganic Hybrid Lead Iodide Perovskite Single Crystals. Adv. Funct. Mater. 2015, 25, 2378-2385.

(29) Schuck, G.; Tobbens, D. M.; Koch-Muller, M.; Efthimiopoulos, I.; Schorr, S. Infrared Spectroscopic Study of Vibrational Modes Across the Orthorhombic-tetragonal Phase Transition in Methylammonium Lead Halide Single Crystals. J. Phys. Chem. C 2018, 122, 5227-5237. 
(30) Phuong, L. Q.; Nakaike, Y.; Wakamiya, A.; Kanemitsu, Y. Free Excitons and Exciton-phonon Coupling in $\mathrm{CH}_{3} \mathrm{NH}_{3} \mathrm{PbI}_{3}$ Single Crystals Revealed by Photocurrent and Photoluminescence Measurements at Low Temperatures. J. Phys. Chem. Lett. 2016, 7, 4905-4910.

(31) Schötz, K.; Askar, A. M.; Peng, W.; Seeberger, D.; Gujar, T. P.; Thelakkat, M.; Köhler, A.; Huettner, S.; Bakr, O. M.; Shankar, K.; et al. Double Peak Emission in Lead Halide Perovskites by Selfabsorption. J. Mater. Chem. C 2020, 8, 2289-2300.

(32) Schötz, K.; Askar, A. M.; Köhler, A.; Shankar, K.; Panzer, F. Investigating the Tetragonal-to-orthorhombic Phase Transition of Methylammonium Lead Iodide Single Crystals by Detailed Photoluminescence Analysis. Adv. Opt. Mater. 2020, 8, 2000455.

(33) Quarti, C.; Mosconi, E.; Ball, J. M.; D’Innocenzo, V.; Tao, C.; Pathak, S.; Snaith, H. J.; Petrozza, A.; De Angelis, F. Structural and Optical Properties of Methylammonium Lead Iodide Across the Tetragonal to Cubic Phase Transition: Implications for Perovskite Solar Cells. Energy Environ. Sci. 2016, 9, 155-163.

(34) TiberCAD Simulation Package; http://www.tibercad.org.

(35) Giannozzi, P.; Baroni, S.; Bonini, N.; Calandra, M.; Car, R.; Cavazzoni, C.; Ceresoli, D.; Chiarotti, G. L.; Cococcioni, M.; Dabo, I.; et al. Quantum Espresso: a Modular and Opensource Software Project for Quantum Simulations of Materials. J. Phys.: Condens. Matter 2009, 21, 395502.

(36) Perdew, J. P.; Burke, K.; Ernzerhof, M. Generalized Gradient Approximation Made Simple. Phys. Rev. Lett. 1996, 77, 3865.

(37) Boyer-Richard, S.; Katan, C.; Traore, B.; Scholz, R.; Jancu, J.M.; Even, J. Symmetry-based Tight Binding Modeling of Halide Perovskite Semiconductors. J. Phys. Chem. Lett. 2016, 7, 3833-3840.

(38) Even, J.; Pedesseau, L.; Jancu, J.-M.; Katan, C. Importance of Spin-Orbit Coupling in Hybrid Organic/inorganic Perovskites for Photovoltaic Applications. J. Phys. Chem. Lett. 2013, 4, 2999-3005.

(39) Umari, P.; Mosconi, E.; De Angelis, F. Relativistic GW Calculations on $\mathrm{CH}_{3} \mathrm{NH}_{3} \mathrm{PbI}_{3}$ and $\mathrm{CH}_{3} \mathrm{NH}_{3} \mathrm{SnI}_{3}$ Perovskites for Solar Cell Applications. Sci. Rep. 2015, 4, 4467.

(40) Kennedy, J.; Eberhart, R. Particle Swarm Optimization. International Conference on Neural Networks 1995, 4, 1942-1948.

(41) Weston, L.; Tailor, H.; Krishnaswamy, K.; Bjaalie, L.; Van de Walle, C. G. Accurate and Efficient Band-offset Calculations from Density Functional Theory. Comput. Mater. Sci. 2018, 151, 174-180. 